\title{
Serum Hepcidin-25 and All-Cause Mortality in Patients Undergoing Maintenance Hemodialysis
}

\author{
Lu-Xi Zou iD \\ Ling Sun (iD) ${ }^{2}$ \\ Rui-Xue Hua ${ }^{3}$ \\ Yu $\mathrm{Wu}^{3}$ \\ 'School of Management, Xuzhou Medical \\ University, Xuzhou, Jiangsu, People's \\ Republic of China; ${ }^{2}$ Department of \\ Nephrology, Xuzhou Central Hospital, \\ Xuzhou Medical University, Xuzhou, \\ Jiangsu, People's Republic of China; \\ ${ }^{3}$ XuZhou Clinical School of Xuzhou \\ Medical University, Xuzhou, Jiangsu, \\ People's Republic of China
}

Correspondence: Ling Sun Department of Nephrology, Xuzhou Central Hospital, Xuzhou Medical University, No. 199, Jiefang South Road, Xuzhou, Jiangsu, 221009, People's Republic of China

Tel +86516 83956891

Fax +8651683840486

Email slpku@।63.com
Background: Hepcidin plays an important role in iron homeostasis, inhibits intestinal iron absorption and iron release from hepatocytes and macrophages, while its clinical utility remained unclear. This study aimed to investigate the associations between hepcidin- 25 and mortality in MHD patients.

Methods: This was a prospective observational cohort of 161 MHD patients, with 2-year follow-up. We investigated the relationships between the variables in our dataset, including serum hepcidin-25, demographic characteristics as well as other clinical parameters.

Results: The median value of baseline serum hepcidin-25 was $31.0(12.1,57.3) \mathrm{ng} / \mathrm{mL}$; therefore, the patients were stratified into two groups (low-level hepcidin-25 group, and highlevel hepcidin-25 group). The serum iron, serum ferritin, transferrin saturation (TSAT), and hsCRP were higher, pre-dialysis creatinine and albumin were lower, and the scores of healthrelated qualities of life were worse in the high-level hepcidin-25 group than in the low-level hepcidin-25 group. Maximal information-based nonparametric exploration analysis suggested that serum hepcidin-25 was associated with ferritin, TSAT, and all-cause mortality. The patients with hepcidin- $25<31 \mathrm{ng} / \mathrm{mL}$ had better survival outcomes than those with hepcidin-25 $\geq 31 \mathrm{ng} / \mathrm{mL}$ during the 24-month follow-up (Log rank test, $\mathrm{P}=0.0017$ ). For per $10 \mathrm{ng} / \mathrm{mL}$ increase of serum hepcidin-25, the hazard ratio (HR) for all-cause mortality was 1.225 (95\% confidence interval [CI]1.085-1.382, $\mathrm{P}<0.001$ ), which remained significant after multivariate adjustments.

Conclusion: Serum hepcidin-25 was associated with ferritin and TSAT, and could be an independent predictor for all-cause mortality in MHD patients. Further research with larger sample size and longer-term follow-up is still needed.

Keywords: hepcidin, mortality, hemodialysis, survival analysis, ESRD

\section{Introduction}

Anemia is mainly caused by decreased production of erythropoietin and increased loss of red blood cell in patients with end-stage renal disease (ESRD). Iron-deficient usually co-existed in the patients undergoing maintenance hemodialysis (MHD). ${ }^{1}$ Iron homeostasis is maintained by absorption of dietary iron in duodenum making up for daily iron loss. In patients receiving MHD, increased blood losses and compromised gastrointestinal iron absorption result in absolute iron deficiency. While reticuloendothelial cell blocks the delivery of its storage iron to marrow for erythropoiesis, which causes functional iron deficiency. ${ }^{2}$

Hepcidin, encoded by the HAMP gene, is a key regulator of iron utilization, which inhibits intestinal iron absorption and iron release from hepatocytes and macrophages. ${ }^{3}$ The promoter of hepcidin contains several binding sites for hypoxia-inducible factors 
(HIFs), therefore, hepcidin could be down-regulated by hypoxia and HIF stabilization. ${ }^{4}$ Previous studies demonstrated that serum hepcidin was reduced in patients who received the HIF stabilizer roxadustat. ${ }^{5,6}$ In addition, hepcidin could also be affected by iron stores, erythropoiesis, inflammation, as well as decreased renal clearance. ${ }^{3,7}$ Hepcidin is an 84 -amino acid prepropeptide, and usually cleaved into three peptide types, hepcidin-20, hepcidin-22, and hepcidin-25, of which, hepcidin-25 is the active form and plays important roles in regulating functional iron deficiency. ${ }^{8}$

Previous studies demonstrated that hepcidin-25 could help to evaluate the iron status and anemia, ${ }^{9,10}$ and participated in the pathophysiology of atherosclerosis and cardiovascular events in patients receiving MHD. ${ }^{11,12}$ However, one study of 56 patients receiving MHD showed that serum hepcidin- 25 could not predict the hematopoietic response to the therapy of intravenous iron plus erythropoiesis-stimulating agent (ESA). ${ }^{13} \mathrm{~A}$ similar conclusion was reported in another study of 61 patients with nondialysis chronic kidney disease (CKD).${ }^{14}$ One study of 50 patients receiving MHD suggested that hepcidin-25 was not related to mortality in the 12-month follow-up. ${ }^{15}$ All the above findings were based on studies of small sample size and short-term follow-up. Therefore, the clinical utility of hepcidin-25 remained uncertain, this study aimed to investigate the associations between hepcidin-25 and mortality in patients receiving MHD.

\section{Materials and Methods}

\section{Participants}

This was a prospective observational study in the clinically stable patients receiving MHD at the Xuzhou Central Hospital (Xuzhou, China). Inclusion criteria: 1) patients with ESRD; 2) aged 18-80 years; 3) duration of dialysis treatment $\geq 3$ months. Exclusion criteria: 1) had infection, inflammation, or malignant diseases; 2) hospital admission for any cause within the preceding 3 months; 3 ) planned to receive kidney transplant or transfer to other facilities in 2 years. The cohort was established in January 2016. All patients were followed up until death or the end date of the study (December 2017). This study followed the International Conference on Harmonized guidelines for good clinical practice and was conducted in accordance with the Helsinki Declaration. The agreement was approved by the Ethics Committee of Xuzhou Central Hospital.

\section{Sample Size Estimation}

To ensure that the sample size was sufficient, the formula was as following:

$$
N=Z_{1-\frac{\alpha}{2}}^{2} p(1-p) / d^{2}
$$

Assuming $\alpha$ value of $0.05, Z_{1-\alpha / 2}$ value of $1.96, d$ value of 0.1 , and $\mathrm{p}$ is set as 0.5 , therefore, $\mathrm{N}=96$. Considering the design effect as 1-3 and the drop-out rate as $10 \%$, the sample size should be from 107 (if design effect=1) to 320 (if design effect $=3$ ). We calculated that this study needed to enroll at least 107 participants.

\section{Data Collection and Measurements}

Baseline demographic and clinical parameters were recorded, including age, gender, body mass index (BMI), etiology of ESRD, comorbidities, and laboratory measures. Questionnaires of the 36-Item Short-Form Health Survey (SF-36) and the Pittsburgh Sleep Quality Index (PSQI) were self-administered to all patients. The plasma and serum were centrifuged and frozen at $-80^{\circ} \mathrm{C}$ until laboratory measurements were made in a certified laboratory (Dian Diagnostics, Nanjing, China). The single-pool Kt/V (spKt/ V) was calculated by two-point urea modeling based on the intradialytic reduction of blood urea and weight loss. ${ }^{16}$ The ESA responsiveness (ERI) was determined by the ratio of weekly ESA dose to hemoglobin $(\mathrm{Hb}) .{ }^{17}$ The duration of dialysis treatment was defined as the time since dialysis was initiated. Serum hepcidin-25 was measured using competitive enzyme-linked immunosorbent assay kits ${ }^{18}$ (Cat. CSB E14239h, Cusabio, China), with a coefficient of variation (CV) $<10 \%$ in both intra- and inter-assay precision analyses.

\section{Statistical Analysis}

Patients' baseline demographic and clinical characteristics, as well as laboratory parameters, were summarized as proportions, mean $( \pm \mathrm{SD})$, or median (interquartile range), and analyzed using one-way analysis of variance, Fisher exact test, and Kruskal-Wallis test depending on the data type. We applied the maximal information-based nonparametric exploration (MINE) statistics, and its maximal information coefficient (MIC) with scores, roughly equal to the coefficient of determination $\left(\mathrm{R}^{2}\right)$ in identifying the relationships between variables in large datasets, ${ }^{19}$ were applied to identify novel relationships using $\mathrm{R}$ software, version 3.4.3 (https://www.r-project.org). Non-parametric Kaplan-Meier plot was used to evaluate the effect of hepcidin-25 on predicting all-cause mortality. Multivariate Cox proportional 
hazard models were performed to identify risk factors for all-cause mortality, and results were expressed as a hazard ratio (HR) for all-cause mortality with per $10 \mathrm{ng} / \mathrm{mL}$ increase of serum hepcidin-25, with $95 \%$ confidence interval (CI). Cox proportional hazard regression model based on restricted cubic spline was applied to explore the non-linear association between serum hepcidin-25 and mortality, adjusted by different confounding factors. A two-sided P-value $<0.05$ was defined as statistically significant. Statistical analyses were performed using the SAS system, version 9.4 (SAS Institute Inc., Cary, NC, USA).

\section{Results}

\section{Baseline Demographics of the Cohort Stratified by Serum Hepcidin-25 Levels}

A total of 161 patients receiving MHD were enrolled in this study with complete data and were assigned to 2 groups by the median value of baseline serum hepcidin25, low-level hepcidin-25, and high-level hepcidin-25 groups (shown in Figure 1). The median hepcidin-25 was $31.0(12.1,57.3) \mathrm{ng} / \mathrm{mL}$. Table 1 showed the baseline characteristics of the cohort, comparing with the lowlevel hepcidin-25 group, the patients in the high-level hepcidin-25 group had older age, higher levels of serum iron, ferritin, TSAT, and hypersensitive C-reactive protein (hs-CRP), lower levels of pre-dialysis creatinine and albumin, as well as worse scores of the SF-36 and PSQI.

\section{Associations Between Hepcidin-25 and Other Variables}

There was a total of 86 variables, involving 9 categories in our dataset. We observed the 216 top-scoring relationships between the 72 variables, with $\mathrm{MIC} \geq 0.3$ (shown in Figure 2). Among them, the hepcidin-25 had strong

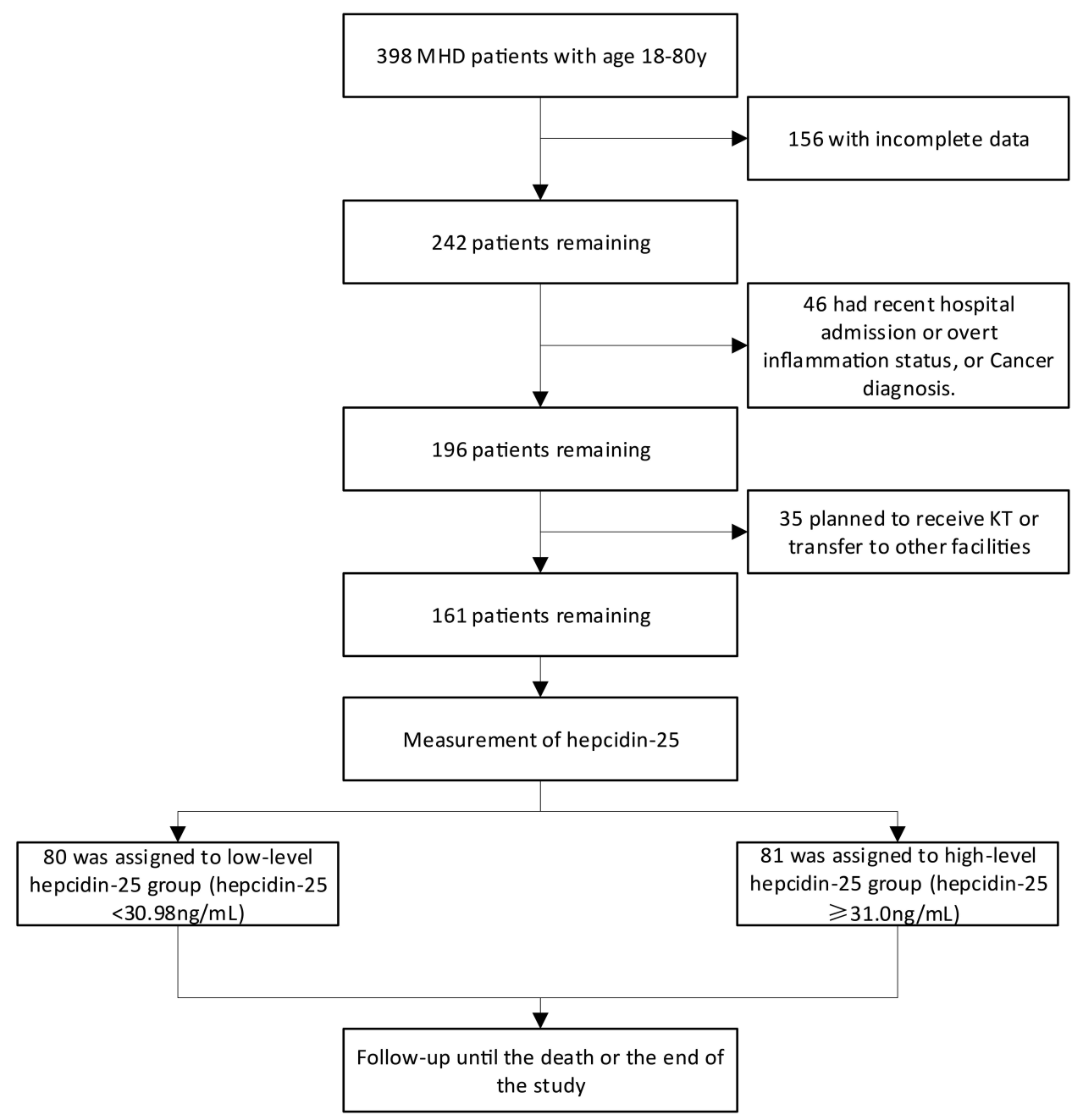

Figure I Flow diagram showing the creation of the main dataset, reasons for exclusions, and assignment according to hepcidin-25 at study entry. Abbreviations: MHD, maintenance hemodialysis; KT, kidney transplant. 
Table I Baseline Characteristics Stratified by Baseline Level of Hepcidin-25 in I6I MHD Patients

\begin{tabular}{|c|c|c|c|c|c|c|c|}
\hline \multirow[b]{3}{*}{$N(\%)$} & \multirow{2}{*}{\multicolumn{2}{|c|}{ Total }} & \multicolumn{4}{|c|}{ Hepcidin-25 (ng/mL) } & \multirow{3}{*}{ P-value } \\
\hline & & & \multicolumn{2}{|l|}{$<31$} & \multicolumn{2}{|l|}{$\geq 31$} & \\
\hline & 161 & $(100.0)$ & 80 & (49.7) & 81 & (50.3) & \\
\hline \multicolumn{8}{|l|}{ Clinical Characteristic } \\
\hline Female, $n(\%)^{a}$ & 68 & $(42.2)$ & 30 & $(37.5)$ & 38.00 & $(46.9)$ & 0.2281 \\
\hline Age, years ${ }^{\mathrm{b}}$ & 52.2 & $(14.9)$ & 48.9 & $(14.4)$ & 55.5 & $(14.7)$ & 0.0047 \\
\hline Catheter, n (\%) & 17 & $(10.6)$ & 1 & $(2.5)$ & 11.0 & $(13.6)$ & 0.1060 \\
\hline Duration of dialysis treatment, months & 50.0 & (32.5) & 51.8 & $(32.3)$ & 48.3 & $(32.8)$ & 0.5002 \\
\hline Dialysis frequency (per week) & 2.5 & $(2,3)$ & 2.5 & $(2,3)$ & 2.5 & $(2,3)$ & 0.4497 \\
\hline HDF frequency (per week) & 1 & $(0.5,1)$ & 1 & $(0.5,1)$ & 1 & $(0.5,1)$ & 0.5185 \\
\hline Body mass index, $\mathrm{kg} / \mathrm{m} 2$ & 21.6 & $(20.1,24.1)$ & 21.8 & $(20.1,25.3)$ & 21.6 & $(19.8,23.6)$ & 0.5294 \\
\hline \multicolumn{8}{|l|}{ Comorbid illnesses } \\
\hline Hypertension, n (\%) & 143 & (88.8) & 73 & (91.3) & 70.00 & (86.4) & 0.3323 \\
\hline Diabetes mellitus, $n$ (\%) & 34 & $(21.1)$ & 14 & $(17.5)$ & 20.00 & $(24.7)$ & 0.1331 \\
\hline Gastrointestinal bleeding, n (\%) & 18 & $(11.2)$ & 4 & $(10.0)$ & 11.00 & $(13.6)$ & 0.3323 \\
\hline \multicolumn{8}{|l|}{ Laboratory data } \\
\hline Hemoglobin (g/dL) & 99.3 & (20.6) & 100.8 & (19.4) & 97.9 & (21.7) & 0.3667 \\
\hline Ferritin $(\mathrm{ng} / \mathrm{mL})$ & 152.3 & $(58.9,987.2)$ & 103.9 & $(56.4,180.8)$ & 580.8 & $(152.3,3721)$ & $<0.0001$ \\
\hline TSAT (\%) & 30.6 & $(20.3,46.2)$ & 28.1 & $(17.3,39.7)$ & 43.6 & $(22,59.4)$ & $<0.0001$ \\
\hline Serum iron (umol/L) & 12.6 & $(9.2,17.6)$ & 11.7 & $(8.3,17.0)$ & 15.0 & $(9.8,21.3)$ & 0.0011 \\
\hline ERI (U/kg/week/g/dL) & 13.0 & $(8.5,16.9)$ & 12.0 & $(7.8,16.4)$ & 13.2 & $(9.7,17.0)$ & 0.1556 \\
\hline Vitamin B/2 (ng/L) & 586 & $(353,2000)$ & 548 & $(359,2000)$ & 592 & $(353,2000)$ & 0.1682 \\
\hline Folic acid (ug/L) & 4.6 & $(2.8,6.5)$ & 4.8 & $(2.8,6.5)$ & 4.5 & $(2.8,6.5)$ & 0.4142 \\
\hline $\mathrm{hsCRP}(\mathrm{mg} / \mathrm{dL})$ & 1.6 & $(0.7,4.2)$ & 1.2 & $(0.5,2.2)$ & 2.7 & $(1.5,6.3)$ & $<0.0001$ \\
\hline Predialysis Creatinine $(\mathrm{mg} / \mathrm{dL})$ & 974 & (349) & 1073 & (362) & 877 & (308) & 0.0003 \\
\hline Albumin (g/dL) & 38.7 & (3.7) & 39.1 & (3.5) & 37.7 & $(3.8)$ & 0.0408 \\
\hline Calcium (mg/dL) & 2.3 & $(2.2,2.4)$ & 2.3 & $(2.2,2.5)$ & 2.3 & $(2.2,2.4)$ & 0.2279 \\
\hline Intact PTH (pg/mL) & 346 & $(156,476)$ & 347.0 & $(198,510)$ & 331 & $(106,45 I)$ & 0.1074 \\
\hline Fasting glucose (mmol/L) & 5.3 & $(4.4,5.3)$ & 5.3 & $(4.5,5.3)$ & 5.3 & $(4.4,5.3)$ & 0.3941 \\
\hline LDL-C (mg/dL) & 2.3 & $(2.2,2.4)$ & 2.3 & $(2.3,2.4)$ & 2.3 & $(1.7,2.4)$ & 0.0691 \\
\hline Potassium (mmol/L) & 5.1 & $(0.8)$ & 5.2 & $(0.8)$ & 5.0 & $(0.8)$ & 0.0656 \\
\hline Magnesium (mmol/L) & 1.2 & $(0.2)$ & 1.2 & $(0.2)$ & 1.2 & $(0.2)$ & 0.2266 \\
\hline Cardiothoracic ratio & 0.5 & $(0.5,0.6)$ & 0.5 & $(0.5,0.6)$ & 0.5 & $(0.5,0.7)$ & 0.1912 \\
\hline Ejection fraction & 0.5 & $(0.5,0.6)$ & 0.6 & $(0.5,0.6)$ & 0.5 & $(0.5,0.6)$ & 0.1137 \\
\hline \multicolumn{8}{|l|}{ 36-Item Short Form Health Survey, SF-36 } \\
\hline Physical Functioning & 55 & $(45,75)$ & 65 & $(50,80)$ & 50 & $(35,70)$ & 0.0009 \\
\hline Role-Physical & 0 & $(0,50)$ & 12.5 & $(0,50)$ & 0 & $(0,25)$ & 0.0143 \\
\hline Pain & 52.0 & $(31,80)$ & 62 & $(41.5,100)$ & 42 & $(31,74)$ & 0.0070 \\
\hline General health & 30.0 & $(15,45)$ & 35 & $(20,46)$ & 20 & $(10,45)$ & 0.0085 \\
\hline Vitality & 55.0 & $(35,75)$ & 55 & $(37.5,77.5)$ & 50 & $(35,65)$ & 0.0149 \\
\hline Social Function & 37.5 & $(12.5,62.5)$ & 43.75 & $(25,75)$ & 37.5 & $(12.5,62.5)$ & 0.0150 \\
\hline Role-Emotional & 0 & $(0,33)$ & 0 & $(0,33)$ & 0 & $(0,33)$ & 0.1421 \\
\hline Mental Health & 60.0 & $(36,80)$ & 68 & $(40,84)$ & 52 & $(36,76)$ & 0.0085 \\
\hline Reported Health Transition & 50.0 & $(25,75)$ & 50 & $(25,75)$ & 50 & $(0,75)$ & 0.0115 \\
\hline \multicolumn{8}{|l|}{ Pittsburgh Sleep Quality Index, PSQI } \\
\hline Sleep quality & 2.0 & $(1,2)$ & 1 & $(1,2)$ & 2 & $(1,2)$ & 0.0221 \\
\hline Sleep latency & 1.0 & $(1,2)$ & 1 & $(1,2)$ & 2 & $(1,2)$ & 0.0246 \\
\hline Sleep duration & 2.0 & $(0,3)$ & 1 & $(0,3)$ & 2 & $(0,3)$ & 0.0479 \\
\hline Habitual sleep efficiency & 2.0 & $(1,2)$ & 1 & $(0.5,2)$ & 2 & $(1,2)$ & 0.0049 \\
\hline Sleep disturbances & 1.0 & $(1,2)$ & 1 & $(1,2)$ & 1 & $(1,2)$ & 0.1293 \\
\hline Use of sleeping medication & 0.0 & $(0,2)$ & 0 & $(0,1.5)$ & 1 & $(0,3)$ & 0.0160 \\
\hline Daytime dysfunction & 2.0 & $(1,3)$ & 2 & $(1,3)$ & 2 & $(1,3)$ & 0.0350 \\
\hline Global PSQI score & 9.0 & $(5,17)$ & 7 & $(5,15)$ & 13 & $(6,17)$ & 0.0023 \\
\hline
\end{tabular}

Notes: ${ }^{a}$ Discrete values expressed as number (percentage). ${ }^{\mathrm{b}}$ Continuous values expressed as means (SD) if normally distributed or median (interquartile range) if skewed. Abbreviations: MHD, maintenance hemodialysis; HDF, hemodiafiltration; TSAT, transferrin saturation; hsCRP, high sensitivity C-reactive protein; ERI, erythropoiesisstimulating agents (ESA) resistance index; PTH, parathyroid hormone; LDL-C, low-density lipoprotein cholesterol. 


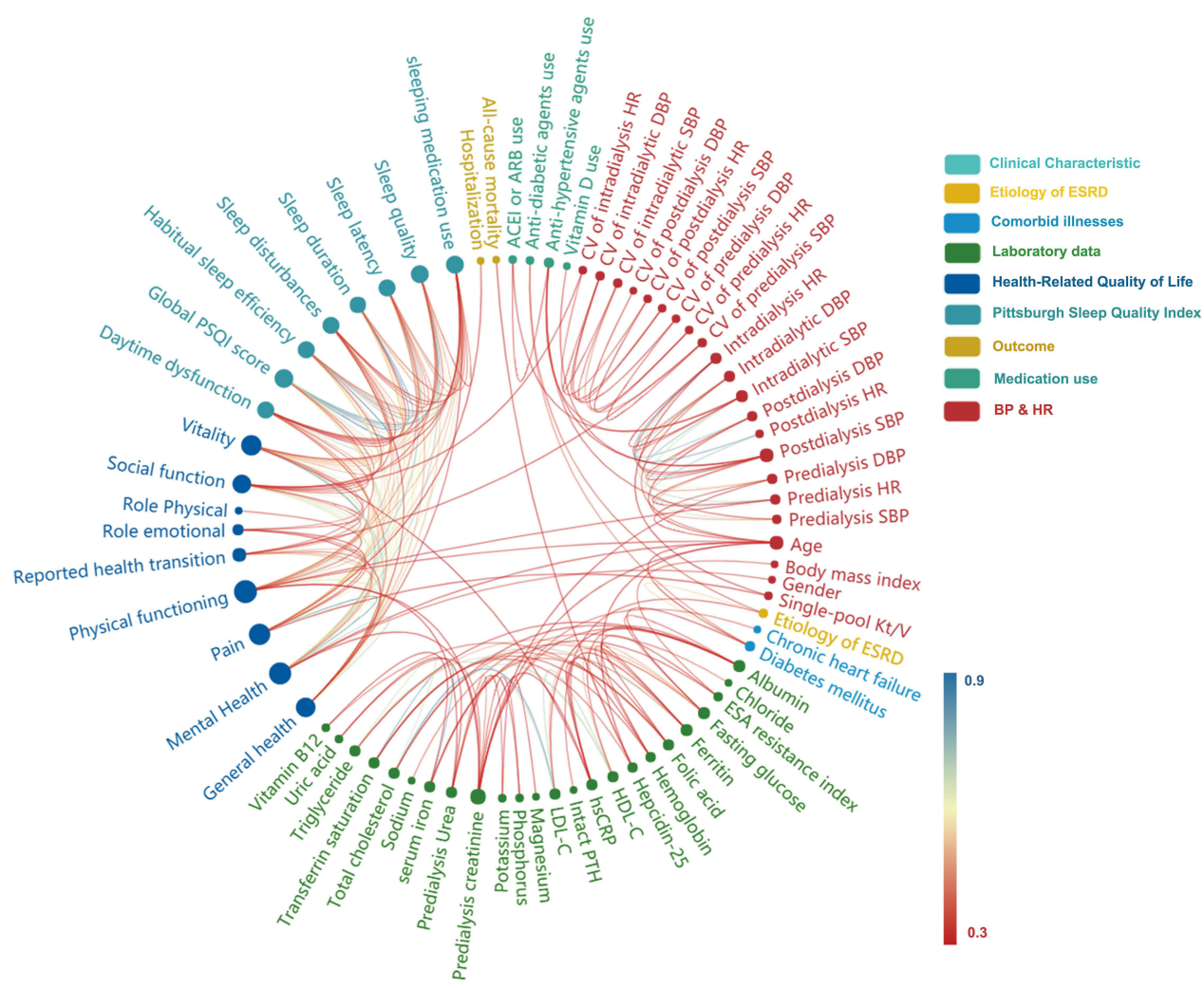

Figure 2 Associations between the variables in the dataset of patients receiving MHD. A topological graph in which nodes correspond to variables and edges correspond to the top 216 relationships. Node size is proportional to the number of these relationships involving the variable. The colors of the edges represent the values of MIC between variables. MIC assigns a perfect score of I to all never-constant noiseless functional relationships, scores that tend to I for a large class of noiseless relationships and a score of 0 to statistically independent variables.

Abbreviations: ESRD, end-stage renal disease; ESA, erythropoietin stimulating agents; hsCRP, high sensitivity C-reactive protein; PTH, parathyroid hormone; LDL-C, lowdensity lipoprotein cholesterol; HDL-C, high-density lipoprotein cholesterol; PSQI, Pittsburgh Sleep Quality Index; ARB, angiotensin II receptor blocker; ACEI, angiotensinconverting enzyme inhibitor; SBP, systolic blood pressure; DBP, diastolic blood pressure; CV, coefficient of variation; HR, heart rate.

associations with ferritin (MIC, 0.46), transferrin saturation (TSAT) (MIC, 0.36), age (MIC, 0.31), and all-cause mortality (MIC, 0.30).

\section{Hepcidin-25 Levels and Mortality}

The mean follow-up duration was $22.81 \pm 3.7$ months; during this period, 19 deaths $(11.8 \%)$ occurred, of which, 16 death occurred in the high-level hepcidin-25 group. Cerebrovascular events $(21.1 \%$, ischemic stroke, and intracerebral hemorrhage) ranked as the first cause leading to death. Patients with hepcidin- $25<31 \mathrm{ng} / \mathrm{mL}$ had better survival outcomes than those with hepcidin- $25 \geq 31 \mathrm{ng} / \mathrm{mL}$ during the 24-month follow-up (Log rank test, $\mathrm{P}=0.0017$ ) (Figure 3A). Similarly, patients in the high-level hepcidin25 group also had a longer duration of dialysis treatment, which meant longer intervals from the first dialysis session to the death or the end of the study (Log rank test, $\mathrm{P}=$ 0.0019) (Figure 3B). Meantime, for per $10 \mathrm{ng} / \mathrm{mL}$ increase of serum hepcidin-25, the unadjusted HR for all-cause mortality was $1.225(95 \%$ CI $1.085-1.382, \mathrm{P}<0.001)$, the HR remained significant after multivariate adjustments (Table 2). Furthermore, restricted cubic spline showed that the curves of adjusted HR for all-cause mortality were relatively stable at hepcidin $-25<31 \mathrm{ng} / \mathrm{mL}$, and then the curves increased significantly at hepcidin- $25 \geq 31 \mathrm{ng} / \mathrm{mL}$, adjusted by age together with hemoglobin (Figure 4A), ferritin (Figure 4B), TSAT (Figure 4C), ERI (Figure 4D), hsCRP (Figure 4E), predialysis creatinine (Figure 4F), albumin (Figure 4G), intact PTH (Figure 4H), respectively.

\section{Discussion}

Iron deficiency is a common cause of anemia in patients receiving MHD. Iron is essential for all living organisms, but iron overload could produce toxic oxidants and cause multiple organ damage. Therefore, iron supplementation is a doubleedged sword and should be managed carefully to achieve the guideline-recommended target of hemoglobin and avoid its side effects. ${ }^{20}$ Recent guidelines recommend that iron status should be monitored periodically through $\mathrm{Hb}$, TSAT, serum ferritin, and hs-CRP. ${ }^{21}$ However, none of them is specific or 

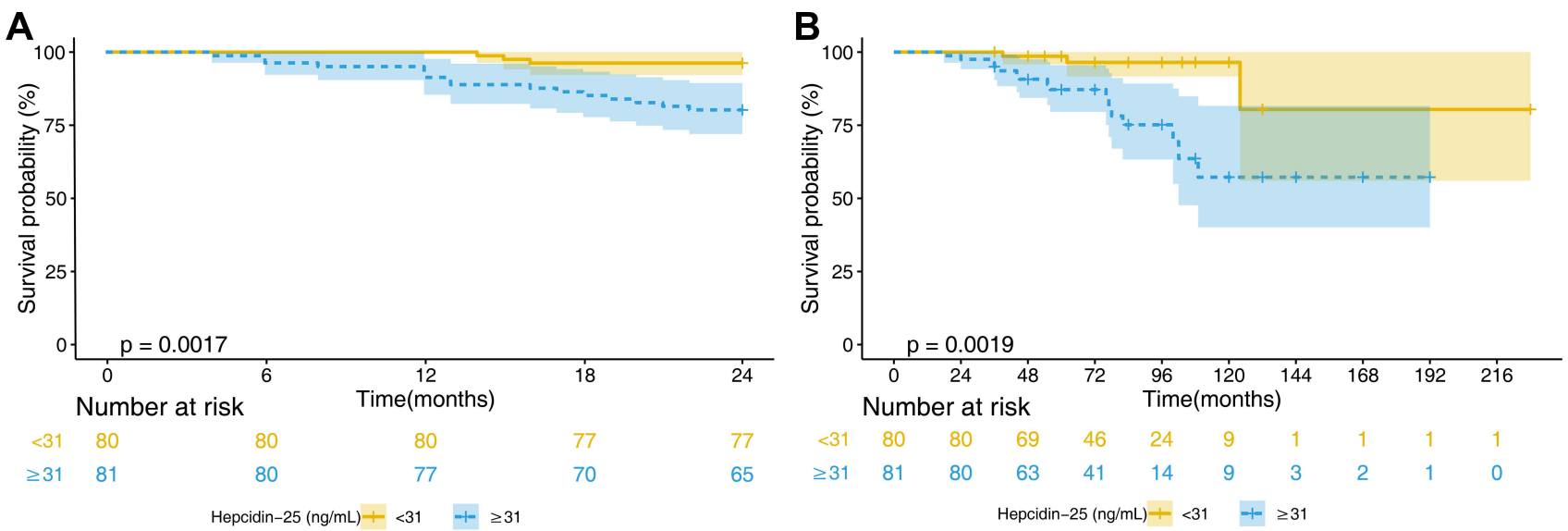

Figure 3 Kaplan-Meier curve of overall survival. The patients receiving MHD were classified into two groups by the baseline serum hepcidin-25. (A) Patients with hepcidin$25<31 \mathrm{ng} / \mathrm{mL}$ had better survival outcomes than those with hepcidin-25 $\geq 3$ I ng/mL during the 24-month follow-up. (B) Patients with hepcidin-25<3। $\mathrm{ng} / \mathrm{mL}$ had longer duration of dialysis treatment than those with hepcidin- $25 \geq 31 \mathrm{ng} / \mathrm{mL}$ (Log rank test, $\mathrm{P}=0.0019$ ). Here, duration of dialysis treatment was defined as the time since dialysis initiated.

sensitive for the regulation of iron metabolism. Hepcidin-25 could regulate iron metabolism through binding ferroportin, inhibiting iron release from hepatocytes and macrophages, and reducing intestinal iron absorption. ${ }^{10}$ Therefore, hepcidin-25 could be a supplement for evaluating functional iron deficiency to conventional iron indices in patients receiving $\mathrm{MHD}^{22}$

KNOW-CKD study ${ }^{23}$ demonstrated that high hepcidin-25 was associated with anemia in patients with non-dialysis CKD. Serum hepcidin was positively correlated with ferritin but had no relationship with inflammatory cytokines and TSAT. $^{24,25}$ Our results showed that the levels of serum iron, TSAT, serum ferritin, and hsCRP were higher in the high-level hepcidin-25 group, with opposite trends of pre-dialysis creatinine and albumin, indicating malnutrition-inflammation complex syndrome in these patients. The inflammation could promote hepcidin expression through several pathways of inflammatory cytokines, such as IL-6 and IL-1 $\beta$. Then the increased hepcidin-25 could result in functional iron deficiency, and influence iron status. ${ }^{7}$

In addition, we introduced a novel statistical method "maximal information-based nonparametric exploration (MINE)", to identify potential relationships between pairs of variables in our dataset, the higher value of MIC, the stronger associations between the variables. As far as we know, there is no previous authoritative literature that can be referred to, thus we chose 0.3 as the cutoff point of MIC to display the variables that might have associations with each other. Consistent with the results in Table 1, the MINE analysis suggested that the circulating hepcidin-25 was associated with ferritin, TSAT, and all-cause mortality.
The restricted cubic spline is an important method for multivariate survival analysis to reveal nonlinear relationships. $^{26}$ Considering the nonlinear associations $^{2}$ between variables in our dataset, the application of restricted cubic spline in the Cox proportional hazard regression model was more suitable than the typical Cox proportional hazard regression model. Therefore, the restricted cubic spline was applied to explore the adjusted non-linear associations between serum hepcidin-25 and mortality, the adjusted HR of hepcidin-25 for mortality showed nonlinear upward trends. The results of these two statistical methods were similar, showing that the risk of mortality increased with the growth of serum levels of hepcidin-25, and the increasing trends were more obvious in higher hepcidin-25 level groups.

\section{Limitations and Strengths}

This study has strengths. First, this is the first attempt of using MINE in clinical medical research until now, the MINE analysis can detect not only the linear but also nonlinear novel relationships between variables in a large dataset, ${ }^{19}$ therefore, it could have a wider application than the traditional analysis, such as Pearson and Spearman correlation coefficient. Next, there was only one published literature of 50 patients receiving MHD reported the correlation between serum hepcidin-25 and mortality, which showed that hepcidin-25 was not related to mortality during 12 months of follow-up. ${ }^{15}$ Our study enrolled 161 patients receiving MHD with 24 months of follow-up and found that high serum hepcidin-25 was associated with all-cause mortality risk. 
Table 2 Hazards Ratio (HR) and Predictors of All-Cause Mortality in Multivariate Cox Proportional Hazard Models

\begin{tabular}{|c|c|c|c|c|c|c|c|c|c|}
\hline & Unadjusted & Adjusted & Adjusted & Adjusted & Adjusted & Adjusted & Adjusted & Adjusted & Adjusted \\
\hline & HR & HR & HR & HR & HR & HR & HR & HR & HR \\
\hline & $(95 \% \mathrm{Cl})$ & $(95 \% \mathrm{Cl})$ & $(95 \% \mathrm{Cl})$ & $(95 \% \mathrm{Cl})$ & $(95 \% \mathrm{Cl})$ & $(95 \% \mathrm{Cl})$ & $(95 \% \mathrm{Cl})$ & $(95 \% \mathrm{Cl})$ & $(95 \% \mathrm{Cl})$ \\
\hline \multirow[t]{2}{*}{ Hepcidin (ng/mL) } & $1.225 * * *$ & $1.262^{*}$ & $1.276 * *$ & $1.294 * *$ & $1.220^{*}$ & $1.22 I^{*}$ & $1.253^{*}$ & $1.243^{*}$ & $1.247 * *$ \\
\hline & $\begin{array}{l}(1.085- \\
1.382)\end{array}$ & $\begin{array}{l}(1.049- \\
1.517)\end{array}$ & $\begin{array}{l}(1.067- \\
1.526)\end{array}$ & $\begin{array}{l}(1.080- \\
1.549)\end{array}$ & $\begin{array}{l}(1.025- \\
1.453)\end{array}$ & $\begin{array}{l}(1.018- \\
1.465)\end{array}$ & $\begin{array}{l}(1.055- \\
1.489)\end{array}$ & $\begin{array}{l}(1.048- \\
1.474)\end{array}$ & $\begin{array}{l}(1.072- \\
1.45 I)\end{array}$ \\
\hline \multirow[t]{2}{*}{ Age } & & $1.062^{* *}$ & $1.073^{* * * *}$ & $1.07^{* * * *}$ & $1.06 * *$ & $1.064 * *$ & $1.070^{* *}$ & $1.066 * *$ & $1.05 * *$ \\
\hline & & $\begin{array}{l}(1.023- \\
1.103)\end{array}$ & $\begin{array}{l}(1.033- \\
1.115)\end{array}$ & $\begin{array}{l}(1.031- \\
I .11)\end{array}$ & $\begin{array}{l}(1.02- \\
1.101)\end{array}$ & $\begin{array}{l}(1.021- \\
1.108)\end{array}$ & $\begin{array}{l}(1.026- \\
1.117)\end{array}$ & $\begin{array}{l}(1.02- \\
1.114)\end{array}$ & $\begin{array}{l}(1.01- \\
1.093)\end{array}$ \\
\hline \multirow[t]{2}{*}{ Hemoglobin ( $\mathrm{g} / \mathrm{dL})$} & & 0.982 & & & & & & & \\
\hline & & $\begin{array}{l}(0.962- \\
1.003)\end{array}$ & & & & & & & \\
\hline \multirow[t]{2}{*}{ Ferritin $(\mathrm{ng} / \mathrm{mL})$} & & & 1.000 & & & & & & \\
\hline & & & $\begin{array}{l}(1.000- \\
1.000)\end{array}$ & & & & & & \\
\hline \multirow[t]{2}{*}{ TSAT (\%) } & & & & 0.993 & & & & & \\
\hline & & & & $\begin{array}{l}(0.975- \\
1.012)\end{array}$ & & & & & \\
\hline \multirow[t]{2}{*}{ ERI (U/kg/week/g/dL) } & & & & & 1.037 & & & & \\
\hline & & & & & $\begin{array}{l}(0.983- \\
1.095)\end{array}$ & & & & \\
\hline \multirow[t]{2}{*}{ hsCRP (mg/dL) } & & & & & & 1.024 & & & \\
\hline & & & & & & $\begin{array}{l}(0.957- \\
1.094)\end{array}$ & & & \\
\hline \multirow{2}{*}{$\begin{array}{l}\text { Predialysis Creatinine } \\
(\mathrm{mg} / \mathrm{dL})\end{array}$} & & & & & & & 1.000 & & \\
\hline & & & & & & & $\begin{array}{l}(0.998- \\
1.002)\end{array}$ & & \\
\hline \multirow[t]{2}{*}{ Albumin (g/dL) } & & & & & & & & 0.977 & \\
\hline & & & & & & & & $\begin{array}{l}(0.849- \\
1.124)\end{array}$ & \\
\hline \multirow[t]{2}{*}{ Intact PTH (pg/mL) } & & & & & & & & & 0.997 \\
\hline & & & & & & & & & $\begin{array}{l}(0.994- \\
1.001)\end{array}$ \\
\hline
\end{tabular}

Notes: $* \mathrm{P}<0.05, * * \mathrm{P}<0.01, * * * \mathrm{P}<0.001$

Abbreviations: TSAT, transferrin saturation; ERI, erythropoiesis-stimulating agents (ESA) resistance index; hsCRP, high sensitivity C-reactive protein; PTH, parathyroid hormone.

This study had limitations. This was a single-center study, therefore, the selection of patients could have introduced bias. We excluded unstable MHD patients, the hepcidin expression could be regulated by multiple factors, such as acute inflammation, therefore, the conclusions here are not applicable for unstable MHD patients, especially 

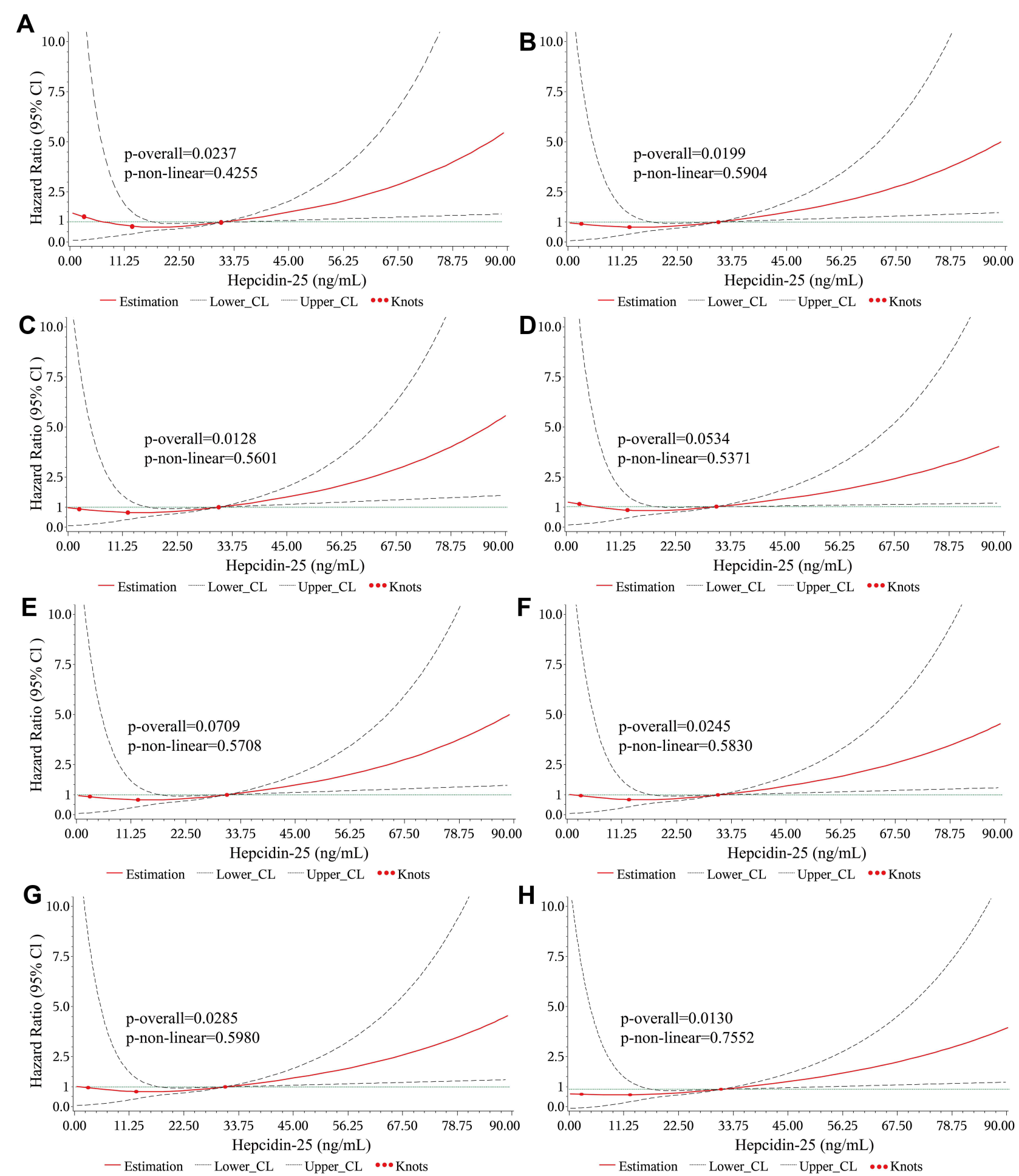

Figure 4 Association between hepcidin-25 and hazard ratio (HR) of all-cause mortality using restricted cubic spline, allowing for non-linear effects, with $95 \%$ confidence intervals (Cls). The reference hepcidin-25 for these plots (with HR fixed as 1.0 ) was $31 \mathrm{ng} / \mathrm{mL}$. Cox regression models were adjusted for age together with hemoglobin (A), ferritin (B), TSAT (C), ERI (D), hsCRP (E), predialysis creatinine (F), albumin (G), intact PTH (H), respectively.

Abbreviations: TSAT, transferrin saturation; ERI, erythropoiesis-stimulating agents (ESA) resistance index; hsCRP, high sensitivity C-reactive protein; PTH, parathyroid hormone. 
the patients with infection, inflammation, malignant diseases. There were very few events of all-cause mortality, and we had to perform the Cox proportional hazard models with potential confounding factors separately. However, to our knowledge, this study had the largest sample size and longest follow-up to investigate the associations between hepcidin-25 and mortality, thus these limitations may be acceptable.

In summary, serum hepcidin-25 was associated with ferritin and TSAT, and could be an independent predictor for all-cause mortality in patients receiving MHD. Monitoring hepcidin-25 could be helpful in the forecast of the survival prognosis in patients receiving MHD. Further research with larger sample size and longer-term follows up was needed.

\section{Ethics and Consent}

The study involved Human Participants and it was performed at the Xuzhou Central Hospital. The study adhered to the International Conference on Harmonization guidelines for Good Clinical Practice and was conducted in accordance with the Declaration of Helsinki. The protocol was approved by the ethical committee of the Xuzhou Central Hospital (Approval No. ZXXY-LJ-20150115001). All participants provided written informed consent.

\section{Funding}

This study was supported by the National Natural Science Foundation of China (81600540), the Science and Technology Foundation of Xuzhou City (KC20182), Science and Technology Foundation of Xuzhou Health Committee (XWKYHT20200020).

\section{Disclosure}

The authors have no conflicts of interest to declare.

\section{References}

1. STAFF N-KGD. KDIGO clinical practice guideline for anemia in chronic kidney disease. Kidney Int Suppl. 2012;2(4):279-335.

2. Goodnough LT, Nemeth E, Ganz T. Detection, evaluation, and management of iron-restricted erythropoiesis. Blood. 2010;116 (23):4754-4761. doi:10.1182/blood-2010-05-286260

3. Agarwal AK, Yee J. Hepcidin. Adv Chronic Kidney D. 2019;26 (4):298-305. doi:10.1053/j.ackd.2019.04.005

4. Malyszko J, Malyszko JS, Matuszkiewicz-Rowinska J. Hepcidin as a therapeutic target for anemia and inflammation associated with chronic kidney disease. Expert Opin Ther Tar. 2019;23(5):407-421. doi:10.1080/14728222.2019.1599358

5. Chen N, Hao C, Peng X, et al. Roxadustat for anemia in patients with kidney disease not receiving dialysis. New Engl J Med. 2019;381 (11):1001-1010. doi:10.1056/NEJMoa1813599
6. Chen N, Hao C, Liu BC, et al. Roxadustat treatment for anemia in patients undergoing long-term dialysis. New Engl J Med. 2019;381 (11):1011-1022. doi:10.1056/NEJMoa1901713

7. Ueda N, Takasawa K. Impact of inflammation on ferritin, hepcidin and the management of iron deficiency anemia in chronic kidney disease. Nutrients. 2018;10(9):1173. doi:10.3390/nu10091173

8. Ganz T, Olbina G, Girelli D, Nemeth E, Westerman M. Immunoassay for human serum hepcidin. Blood. 2008;112(10):4292-4297. doi:10.1182/blood-2008-02-139915

9. Nairz M, Theurl I, Wolf D, Weiss G. Iron deficiency or anemia of inflammation?: differential diagnosis and mechanisms of anemia of inflammation. Wien Med Wochenschr. 2016;166(13-14):411-423. doi:10.1007/s10354-016-0505-7

10. Ueda N, Takasawa K. Role of Hepcidin-25 in Chronic Kidney Disease: anemia and Beyond. Curr Med Chem. 2017;24 (14):1417-1452. doi:10.2174/0929867324666170316120538

11. Alaaddin K, Ozlem Y, Bulent E, et al. Is hepcidin-25 a predictor of atherosclerosis in hemodialysis patients? Hemodial Int. 2016;20:191-197. doi:10.1111/hdi.12355

12. Xu Y, Wang Y, Hu H, Li J, Tian T. Relationship between serum hepcidin levels and cardiovascular disease in patients with maintenance hemodialysis. Physiol Int. 2020;107(4):491-500. doi:10.1556/ 2060.2020 .00040

13. Tessitore N, Girelli D, Campostrini N, et al. Hepcidin is not useful as a biomarker for iron needs in haemodialysis patients on maintenance erythropoiesis-stimulating agents. Nephrol Dial Transplant. 2010;25 (12):3996-4002. doi:10.1093/ndt/gfq321

14. Gaillard CA, Bock AH, Carrera F, et al. Hepcidin response to iron therapy in patients with non-dialysis dependent CKD: an analysis of the FIND-CKD trial. PLoS One. 2016;11(6):e0157063. doi:10.1371/ journal.pone. 0157063

15. Petruliene K, Ziginskiene E, Kuzminskis V, Nedzelskiene I, Bumblyte IA. Hepcidin serum levels and resistance to recombinant human erythropoietin therapy in hemodialysis patients. Medicina (Kaunas). 2017;53(2):90-100. doi:10.1016/j.medici.2017.03.001

16. Daugirdas JT. Kt/V (and especially its modifications) remains a useful measure of hemodialysis dose. Kidney Int. 2015;88 (3):466-473. doi:10.1038/ki.2015.204

17. Suttorp MM, Hoekstra T, Rotmans JI, et al. Erythropoiesisstimulating agent resistance and mortality in hemodialysis and peritoneal dialysis patients. BMC Nephrol. 2013;14:200. doi:10.1186/ 1471-2369-14-200

18. Busbridge M, Griffiths C, Ashby D, et al. Development of a novel immunoassay for the iron regulatory peptide hepcidin. Br J Biomed Sci. 2009;66(3):150-157. doi:10.1080/09674845.2009.11730263

19. Reshef DN, Reshef YA, Finucane HK, et al. Detecting Novel associations in large datasets. Science. 2011;334(6062):1518-1524. doi:10.1126/science. 1205438

20. Slotki I, Cabantchik ZI. The labile side of iron supplementation in CKD. J Am Soc Nephrol. 2015;26(11):2612-2619. doi:10.1681/ ASN.2015010052

21. Mikhail A, Brown C, Williams JA, et al. Renal association clinical practice guideline on Anaemia of Chronic Kidney Disease. BMC Nephrol. 2017;18(1):345. doi:10.1186/s12882-017-0688-1

22. Wojtaszek E, Glogowski T, Malyszko J. Iron and chronic kidney disease: still a challenge. Front Med (Lausanne). 2020;7:565135. doi:10.3389/fmed.2020.565135

23. Lee SW, Kim YH, Chung W, et al. Serum hepcidin and iron indices affect anemia status differently according to the kidney function of non-dialysis chronic kidney disease patients: korean Cohort Study For Outcome in Patients with Chronic Kidney Disease (KNOW-CKD). Kidney Blood Press Res. 2017;42(6):1183-1192. doi: $10.1159 / 000485865$

24. Gaweda AE. Markers of iron status in chronic kidney disease. Hemodial Int. 2017;21(Suppl 1):S21-S27. doi:10.1111/hdi.12556 
25. Ogawa C, Tsuchiya K, Tomosugi N, Kanda F, Maeda K, Maeda T. Low levels of serum ferritin and moderate transferrin saturation lead to adequate hemoglobin levels in hemodialysis patients, retrospective observational study. PLoS One. 2017;12(6):e0179608. doi:10.1371/ journal.pone. 0179608
26. Wei Y, Zhou J, Zhang Z, et al. Application of restricted cube spline in cox regression model. Zhonghua Yufang Yixue Zazhi. 2020;54 (10):1169-1173. doi:10.3760/cma.j.cn112150-20200804-01092

\section{Publish your work in this journal}

The International Journal of General Medicine is an international, peer-reviewed open-access journal that focuses on general and internal medicine, pathogenesis, epidemiology, diagnosis, monitoring and treatment protocols. The journal is characterized by the rapid reporting of reviews, original research and clinical studies across all disease areas. The manuscript management system is completely online and includes a very quick and fair peer-review system, which is all easy to use. Visit http://www.dovepress.com/ testimonials.php to read real quotes from published authors. 